\title{
Overcoming the diffraction limit with high-index dielectric particles
}

\author{
V. Pacheco-Peña ${ }^{1}$, M. Beruete ${ }^{2-3}$ \\ ${ }^{1}$ School of Engineering, Newcastle University, Merz Court, Newcastle Upon Tyne NE17RU, United Kingdom \\ ${ }^{2}$ Antennas Group-TERALAB, Universidad Pública de Navarra, Campus de Arrosadía, 31006 Pamplona, Spain \\ ${ }^{3}$ Institute of Smart Cities (ISC), Public University of Navarra, 31006 Pamplona, Spain \\ victor.pacheco-pena@newcastle.ac.uk, miguel.beruete@unavarra.es
}

\begin{abstract}
In this communication, we propose and demonstrate the ability to produce photonic nanojets using high refractive index dielectric particles immersed in air. An extremely narrow focus is produced just at the output surface of the proposed $2 \mathrm{D}$ and $3 \mathrm{D}$ particles with a spatial resolution of $\sim 0.14 \lambda_{0}$ and $\sim 0.06 \lambda_{0}$, respectively. In addition to the focusing performance, the imaging capabilities is also studied by introducing metallic spheres within the region of the photonic nanojet demonstrating that distances between objects as small as $\sim 0.06 \lambda_{0}$ can be distinguished with the proposed structures.
\end{abstract}

\section{INTRODUCTION}

Overcoming the intrinsic diffraction limit of electromagnetic waves has become a prominent research area for decades in order to improve the spatial resolution of imaging systems. This is due to the fact that the scattered field produced by small features is prominently evanescent. Hence, the detection of such rapidly spatially decaying fields is difficult to achieve [1]. Within this realm, different techniques have been proposed in order to improve imaging systems and overcome the diffraction limit such as metamaterials [2], [3], superoscillatory focusing devices [4], [5] and microspherical dielectrics [6]. Additionally, photonic nanojets (PNJ) were also proposed several years ago (and demonstrated at optical frequencies) in order to produce focal spots with high-intensity and improved spatial resolution [7].

PNJs are non-evanescent and narrow beams produced just at the output surface of a cylinder/sphere (2D/3D) illuminated with a planewave. To produce a PNJ, the refractive index contrast between the particle $(n)$ and the background medium $\left(n_{0}\right)$ should be $n / n_{0}<2$. By doing this, a narrow focus with a resolution in the order of $\lambda_{0} / 3$ can be achieved ( $\lambda_{0}$ as the incident wavelength) [7]. However, one of the issues is that the overall radius of the dielectric particles should be $r>\lambda_{0}$ which may not be suitable for compact applications. Different geometries have been also proposed in order to produce PNJs, in addition to cylinders and spheres, such as gradient index particles [8], cones and ellipsoids [9] and cuboids [10]-[13], to name a few.

Inspired by the opportunities offered by PNJs and the necessity of improving imaging devices for microscopy and sensing applications, in this work we propose an alternative and intriguing technique to produce high-spatial resolution PNJs using high index dielectric particles immersed in vacuum, i.e., high index contrast between the dielectrics and the background medium $\left(n / n_{0}>>2\right)$. With this technique, the Weierstrass formulation for solid immersion lenses [14] is used in the design of 2D/3D dielectric cylinders/spheres with a more compact size $(r=$ $0.55 \lambda_{0}$ ). It will be shown how the resolution of the PNJ can be highly improved with this technique achieving values of up to $\sim 0.06 \lambda_{0}[15]$.

\section{DESIGN AND FOCUSING PERFORMANCE}

To begin with, the schematic representation of the dielectric structure is shown in Fig. 1a. It consists of a dielectric cylinder/sphere immersed in vacuum $\left(n_{0}=1\right)$. Titanium Dioxide $\left(\mathrm{TiO}_{2}\right)$ is used for the dielectric particles because of its high refractive index and low loss at the design frequency of $f_{0}=50 \mathrm{GHz}\left(\lambda_{0}=6 \mathrm{~mm}\right), \operatorname{Re}\{n\} \sim 9.59$ and $\tan \delta \sim 0.05$, respectively. As shown in [15], preliminary studies with the full 2D cylinders demonstrated that the PNJ was produced inside of the dielectric because of the high index contrast $n / n_{0}>>2$ (more details will be provided during the conference). To move the PNJ just at the output surface of the dielectric, the Weierstrass 
formulation for solid immersion lenses [14] is used and the profile of the 2D cylinder is cut at the distance $a$ from its center (see Fig. 1a) using the following simple equation:

$$
a=\left(1+n^{-1}\right) r-r
$$

With this configuration, the numerical results of the power distribution on the $x z(H)$-plane for a $2 \mathrm{D}$ cylinder and 3D sphere are shown in the insets of Fig. 1b,c where a clear PNJ is produced at the output surface of the high index dielectric particles. The numerical results of the intensity enhancement (defined as the ratio between the power distribution with and without the dielectrics) distribution along the transversal $x$ axis is shown in Fig. 1 b,c. As observed an extremely narrow focus is produced by the 2D/3D structures with a Full-Width at Half-Maximum (FWHM $x, y$, defined as the distance along the $x / y$ axis at which the power distribution has decayed half its maximum) of FWHM $x \sim 0.14 \lambda_{0}$ for the $2 \mathrm{D}$ case and FWHM $x \sim 0.08 \lambda_{0}$ and FWHM $y \sim 0.06 \lambda_{0}$ (the small difference observed between both spatial resolutions may be because of the finite number of data points acquired during the calculation which can introduce a small variation of the beam width at the focal position) for the 3D sphere, demonstrating the ability to achieve record resolutions with the proposed technique.
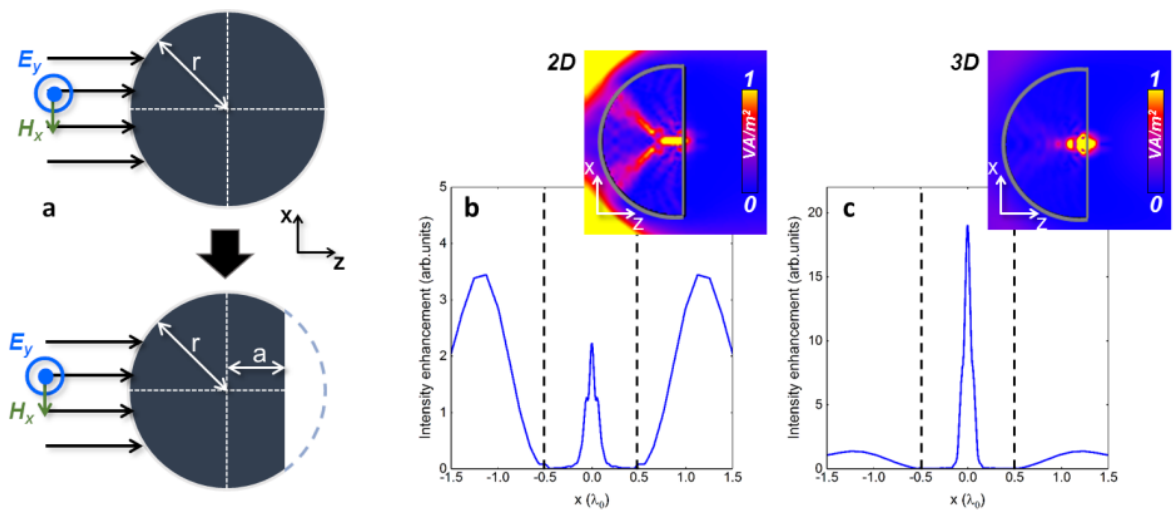

Fig. 1. (a) Schematic representation of the proposed high-index dielectric structure to produce PNJ's. Numerical results of the intensity enhancement along the $x$ axis at the output surface of a 2D cylinder (b) and 3D sphere (c) after applying the design procedure shown in panel a. The power distribution on the $x z$-plane for both $2 \mathrm{D} / 3 \mathrm{D}$ cases are shown as insets $(\mathrm{b}, \mathrm{c})$.

\section{BACKSCATTERING ENHANCEMENT FOR IMAGING}

To further evaluate the performance of the proposed high-index dielectric particles, the imaging capabilities were also studied. For this case, two gold $(\mathrm{Au})$ spheres with a subwavelength diameter $\left(0.067 \lambda_{0}\right)$ were placed just at the output surface of the 3D high-index dielectric sphere and moved along the $x$ and $y$ axes. By doing this, the backscattering enhancement (calculated as the backscattering with and without the spheres) was evaluated (Fig. 2a). As observed, a variation of the scanning-probe technique is used with the dielectric particle as the probe. Different distances between the Au spheres were considered (more details will be provided during the conference).
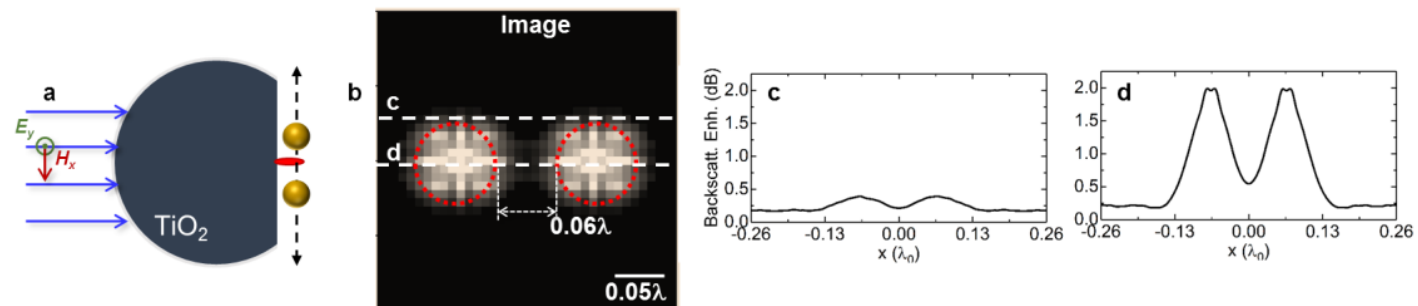

Fig. 2. (a) Schematic representation of the configuration used to evaluate the imaging capabilities of the proposed highindex dielectrics. (b) Results of the backscattering enhancement and image generated considering two Au spheres separated by a subwavelength distance. $(\mathrm{c}, \mathrm{d})$ Backscattering enhancement along the $x$ axis extracted from the dashed lines shown in $\mathrm{b}$. 
As an example, the image generated by the dielectric structure considering two Au spheres separated by a distance of $0.06 \lambda_{0}$ is shown in Fig. $2 b$ (the performance for smaller distances between the spheres will be presented during the conference). A clear image is obtained with a clear gap between both Au spheres. For completeness, the backscattering enhancement along the $x$ axis at the location shown in Fig. $2 \mathrm{~b}$ as dashed lines are shown in Fig. $2 \mathrm{c}, \mathrm{d}$ where again the high spatial resolution achieved with the proposed PNJ is evident.

\section{CONCLUSION}

In this work, a mechanism to generate PNJs with high spatial resolution has been proposed and demonstrated using high-index dielectric particles immersed in vacuum with size comparable to the incident wavelength. The focusing properties of the 2D and 3D dielectric particles have been evaluated demonstrating an extremely high resolution of the focus of $\sim 0.14 \lambda_{0}$ and $\sim 0.06 \lambda_{0}$, respectively. As an application of the proposed structure, the backscattering enhancement and imaging capabilities were evaluated by inserting Au spheres within the PNJ region. A clear image of two Au spheres was demonstrated with the proposed system even with subwavelength distances between the Au particles of $\sim 0.06 \lambda_{0}$. The proposed technique may be applied to sensors and imaging devices where high-spatial resolution and compact structures are needed

\section{ACKNOWLEDGEMENT}

\section{V.P.-P. is supported by the Newcastle University (Newcastle University Research Fellow).}

\section{REFERENCES}

[1] M. Born and E. Wolf, Principles Of Optics, 7th ed. New York: Cambridge University Press, 1999.

[2] N. Fang, H. Lee, C. Sun, and X. Zhang, "Sub-diffraction-limited optical imaging with a silver superlens.," Science, vol. 308, no. 5721, pp. 534-7, Apr. 2005.

[3] V. Pacheco-Peña, N. Engheta, S. A. Kuznetsov, A. Gentselev, and M. Beruete, "Experimental realization of an epsilonnear-zero graded-index metalens at terahertz frequencies,” Phys. Rev. Appl., vol. 8, pp. 034036-1-10, 2017.

[4] E. T. F. Rogers et al., "A super-oscillatory lens optical microscope for subwavelength imaging.," Nat. Mater., vol. 11, no. 5, pp. 432-5, May 2012.

[5] S. Legaria, V. Pacheco-Peña, and M. Beruete, "Super-Oscillatory Metalens at Terahertz for Enhanced Focusing with Reduced Side Lobes," Photonics, vol. 5, no. 4, p. 56, Dec. 2018.

[6] Z. Wang et al., "Optical virtual imaging at $50 \mathrm{~nm}$ lateral resolution with a white-light nanoscope.," Nat. Commun., vol. 2, p. 218, Jan. 2011.

[7] A. Heifetz, S.-C. Kong, A. V Sahakian, A. Taflove, and V. Backman, "Photonic Nanojets.," J. Comput. Theor. Nanosci., vol. 6, no. 9, pp. 1979-1992, Sep. 2009.

[8] Y. E. Geints, A. A. Zemlyanov, and E. K. Panina, "Photonic nanojet calculations in layered radially inhomogeneous micrometer-sized spherical particles," J. Opt. Soc. Am. B, vol. 28, no. 8, p. 1825, Jul. 2011.

[9] C. Y. Liu and F. C. Lin, "Geometric effect on photonic nanojet generated by dielectric microcylinders with non-cylindrical cross-sections," Opt. Commun., vol. 380, pp. 287-296, 2016.

[10] V. Pacheco-Peña, M. Beruete, I. V. Minin, and O. V. Minin, "Terajets produced by dielectric cuboids," Appl. Phys. Lett., vol. 105, no. 8, p. 084102, Aug. 2014.

[11] V. Pacheco-Peña, M. Beruete, I. V Minin, and O. V Minin, "Multifrequency focusing and wide angular scanning of terajets," Opt. Lett., vol. 40, no. 2, pp. 245-248, 2015.

[12] I. V Minin, O. V Minin, V. Pacheco-Peña, and M. Beruete, "Localized photonic jets from flat, three-dimensional dielectric cuboids in the reflection mode," Opt. Lett., vol. 40, no. 10, p. 2329, May 2015.

[13] V. Pacheco-Peña, I. V Minin, O. V Minin, and M. Beruete, "Comprehensive analysis of photonic nanojets in 3D dielectric cuboids excited by surface plasmons," Ann. Phys., vol. 528, no. 9-10, pp. 684-692, Oct. 2016.

[14] S. M. Mansfield and G. S. Kino, "Solid immersion microscope," Appl. Phys. Lett., vol. 57, no. 24, p. 2615, 1990.

[15] V. Pacheco-Peña and M. Beruete, "Photonic nanojets with mesoscale high-index dielectric particles," J. Appl. Phys., vol. 125, no. 8, p. 084104, Feb. 2019. 